\title{
Reasons for dropping out of internet-based problem gambling treatment, and the process of recovery - a qualitative assessment
}

\author{
Anders Nilsson ${ }^{1}\left(\mathbb{1} \cdot\right.$ Olivia Simonsson $^{2} \cdot$ Clara Hellner $^{2}$
}

Accepted: 6 October 2021

(c) The Author(s) 2021

\begin{abstract}
Problem gambling (PG) is a condition affecting the economy, mental health, and relationships of both the individuals with gambling problems, and concerned significant others (CSOs). While PG is treatable, few individuals with gambling problems seek treatment, and many drop out of treatment. This qualitative study aims to investigate a) the reasons for individuals with gambling problems to drop-out from Internet-based PG treatment, and b) what individuals with gambling problems and CSOs find helpful and unhelpful processes in PG recovery. A total of 16 participants (8 individuals with gambling problems and 8 CSOs) who had participated in an Internet-based PG treatment were interviewed over the telephone in a semi-structured interview. The interviews were analyzed using thematic analysis. Drop-out from treatment was one aspect of an overarching theme identified as unstable path to recovery, where alternating periods of progress and setbacks delineate several aspects of PG. Relapses, negative emotions, and changing life circumstances were identified to separately, and in combination, contribute to drop-out. Drop-outs were also explained by participants' experiences of a reduced need for treatment. Openness and a support from CSOs and peers were identified as themes important for recovery. The results suggest that PG treatments should consider the emotional state, and comorbidities of the patients, in order to reduce drop-out and improve chances of recovery.
\end{abstract}

Keywords Gambling $\cdot$ Problem gambling $\cdot$ Drop-out $\cdot$ Concerned significant others $\cdot$ Attrition

\section{Introduction}

Approximately $2 \%$ of the world population have a gambling problem (Williams et al., 2012), and it is estimated that another six people are concerned significant others (CSO) to every individual with a gambling problem (Goodwin et al., 2017). Problem Gambling (PG) is characterized by difficulties in limiting money and/or time spent on gambling which leads to adverse consequences for the gambler, others, or for the community (Neal et al., 2005). The adverse consequences include financial difficulties, strained relationships,

Pre-registration The study was registered at clinicaltrials.gov with number: NCT02543372

Anders Nilsson

anders.nilsson.2@ki.se

1 Centrum För Psykiatriforskning, Norra Stationsgatan 69, 7tr, 11364 Stockholm, Sweden

2 Department of Clinical Neuroscience, Centre for Psychiatry Research, Karolinska Institutet \& Stockholm Health Care Services, Stockholm County Council, Stockholm, Sweden and poor mental and physical health for both the individual who gambles and affected CSOs (Grant et al., 2006; Hodgins et al., 2011; Kalischuk et al., 2006).

A large body of research has investigated the impact of psychological interventions for problem gambling, such as motivational interviewing, 12-step treatments, psychodynamic interventions and cognitive behavioral therapy (CBT) (Potenza et al., 2019; Yakovenko \& Hodgins, 2016). CBT has the most robust evidence supporting its effectiveness (Cowlishaw et al., 2012). However, the high percentage of participants dropping out of treatment limits what conclusions can be drawn from these trials. On average, 39\% have been estimated to drop-out prematurely from PG treatments trials (Pfund et al., 2021), compared to an estimated 23-50\% in outpatient treatment for drug abuse (Brorson et al., 2013), and 20\% for other psychiatric disorders (Swift \& Greenberg, 2012). This poses a substantial threat to the validity of the studies, and what conclusions can be drawn from them, referred to as attrition bias (Westphal, 2007). Attrition usually refers to when participants drop-out of treatment or are lost to follow up by not filling out followup measures in intervention research (Eysenbach, 2005). 
Treatment completers tend to have a better prognosis overall compared to the entire group that started treatment, resulting in a biased interpretation of the results. (Jüni et al., 2001).

While drop-out is a reality for most studies on PG interventions, the phenomena is quite poorly understood. A recent meta-analysis of 24 studies by Pfund et al. (2021) found that increases in the percentage of married participants was associated with lower rates of drop-out, while an earlier meta-analysis by Melville et al. (2007) including 12 RCTs revealed that a long list of factors - such as age, comorbidity, length of PG - were associated with dropout, but no predictor occurred in more than one study. Other trials have indicated that younger age, single marital status, novelty seeking personality (Aragay et al., 2015; Smith et al., 2010), impulsivity (Ramos-Grille et al., 2015), and comorbidity (Pelletier et al., 2008) predict dropout, but also that clients who drop out are no different than other clients (in a study of all female participants) (Dowling, 2009). A study by Ronzitti et al. (2017) found differences between predictors related to pre-treatment dropout (younger age, drug use), and in-treatment dropout predictors (family history of gambling, low levels of PG, smoking), but there is still a general lack of consistency in what predictors are found to correlate with dropout. One explanation is that different definitions have been used for dropout, e.g. not completing all sessions, missing three treatment sessions or more, or discontinuing before achieving reliable change (Pfund et al., 2018). For the purposes of this study, we have chosen to define drop-out as completing less than half of the treatment. We thus include both participants who dropped out before treatment start, and those who have participated in parts of the treatment. This allows us to capture the heterogeneity in the group and get a more comprehensive understanding of the process of dropping out from PG treatments.

Qualitative assessments on reasons for drop-out have found that clients that drop out miss the thrill of gambling (Grant et al., 2004), and that non-compliance with homework, gambling as a way to alter emotional states, and high levels of guilt and stigma might partly explain dropouts (Dunn et al., 2012). Dropout has also been found to be related to changing life circumstances unrelated to gambling, such as clients or therapists moving, or starting a new job (Dunn et al., 2012). Dropout from PG treatment can also be related to recovery; clients may not feel the need to participate in treatment any longer (Brown, 1986; Dunn et al., 2012), contrary to the widely held notion that clients who drop out fare worse than those who continue (Jüni et al., 2001). Treatment drop-out is certainly not limited to PG interventions, and it has been identified as a particular challenge in various Internet-based interventions (Eysenbach, 2005; Christensen et al., 2009; Melville et al., 2010), where therapist contact is generally lower, and the expectation to work independently with home-works and assignments might be higher.

Ultimately, however, drop-out from PG treatments imply that the treatments offered are insufficient in providing adequate support. It could also be seen as an indication that there is a limited understanding of the recovery process in PG, or how to implement our knowledge of recovery when designing treatment protocols. The recovery process in PG is generally a complex, long-term, and often cyclical process that individuals with gambling problems might have to go through several times before reaching what might be viewed as sustainable recovery (Anderson et al, 2009a; Nixon \& Solowoniuk, 2006; Pickering et al., 2020a, b; Slutske, 2014; Williams et al., 2015). Some factors identified as driving the process of change and recovery are financial concerns (Hodgins and el-Guebaly 2000), life changes (Cunningham et al., 2009), family influence and emotional or cognitive reappraisal of gambling (Cunningham et al., 2009; Toneatto et al., 2008; Hodgins and el-Guebaly 2000; Pickering et al., 2020a, b; Vasiliadis \& Thomas, 2018; Rossini-Dib et al., 2015). Given the rates of drop-out, the fact that the vast majority of individuals with gambling problems never seek treatment (Slutske, 2014; Statens folkhälsoinstitut, 2010), and that most individuals with gambling problems recover without formal treatment (Slutske, 2014), much is yet to be learned about the role of formal treatment in PG recovery.

By interviewing individuals with gambling problems who have dropped out from a PG treatment, it is possible to gain a broader understanding on what might explain treatment drop-out, and how the participants view the process of recovery in PG. It is possible to gain a more comprehensive view of drop-out and recovery by also interviewing CSOs of individuals with gambling problems who have dropped out of treatment.

The aim of this study was to investigate the perspective of individuals with gambling problems and CSOs who had participated in a study on Internet-based treatment for PG on a) the reasons for individuals with gambling problems to drop-out of PG treatment, and b) what individuals with gambling problems and CSOs find helpful and unhelpful processes in the recovery of $\mathrm{PG}$ ?

\section{Materials \& Methods}

This study has a qualitative design, and is part of a larger randomized controlled trial (RCT) investigating the effects of involving CSOs in an Internet-based PG treatment (Nilsson et al., 2020). The qualitative approach was chosen because of the explorative nature of the research questions, and since it enabled us to capture individual psychological processes in depth, and thoughts on recovery. 
The in-depth interviews were conducted in Swedish and recorded by Author 1 over telephone between January $24^{\text {th }}$ 2019-May $8^{\text {th }} 2019$, and lasted between $10-25 \mathrm{~min}$. The interviews were conducted approximately two to three years after the participants had dropped out from the RCT, and approximately one to two years after the last follow-up measures were sent out to the participants. There is a risk that this time frame renders various memory biases, but it could be argued that it gives participants a more global understanding of the often cyclical and prolonged nature of PG, providing a "freedom from the past" enabling, as proposed by Nixon and Solowoniuk (2006), enabling us to capture the recovery process over time. The interview guide (see Table 1) was developed by Author 1 and Author 3 and consisted of 16 questions for gamblers and 16 questions for CSOs related to the research questions of this paper. The questions were phrased to be somewhat broader in scope than the research questions, in order to facilitate a better understanding of the situation for the participants. The interviews were semi-structured, which in this instance meant that additional follow-up questions were posed that were not originally part of the interview guide to gain more information. Furthermore, not all questions had to be posed to all participants, if they had already provided the answer at an earlier stage in the interview. Author 1 had previously been involved in all phases of the RCT, but had not participated as a therapist, and hence had had no direct contact with the participants included in this study.

\section{Interventions}

The participants in this study had previously taken part in a randomized controlled Internet-based CBT trial involving both gamblers and CSOs, see the study protocol (Nilsson et al., 2016), the pilot study (Nilsson et al., 2018), and the RCT (Nilsson et al., 2020) for more details. The trial consisted of two arms; the CBT group where only the gambler received treatment modules, and the behavioral couples therapy (BCT) intervention where both the gambler and the CSO received treatment modules. Both treatments contained 10 treatment modules, delivered over a period of 12 weeks, and participants received telephone and email support from a therapist. The therapists were instructed to remind participants through email notifications, text messages, or by calling them should the participants have failed to return module assignments on schedule. A CSO was in the RCT defined as a friend, sibling, child or partner of the gambler, and they had to have known each other for at least three months prior to inclusion in the study. The individual with gambling problems had to fulfill the criteria of gambling problems according to the Problem Gambling Severity Index (PGSI) (Ferris \& Wynne, 2001), while the CSO could display no such symptoms. Both had to be at least 18 years old,

Table 1 Interview guide

Hi!

My name is NN, and I'm contacting you because you've participated in the study "[Name of study]". Now we want to find out more about if and how the treatment worked, to see if it can be of any help for other individuals. For this reason, I wonder if you would have time to answer some questions about your participation in the study? It will take approximately 10-20 min

1. What made you apply for [Name of study] from the very beginning?

2. What was your goal with the treatment?

3. What did you think about the content of the treatment?

a. Possible follow-up question: Did you feel something was missing in the treatment?

4. What did you think about the treatment format, i.e. that the study was delivered online?

5. What did you think about participating in the study together with a CSO/the gambler?

6. What made/do you think made you/him/her discontinue your/his/her participation in the treatment?

7. Could you describe your/his/her gambling during this period?

8 . Was your/his/her gambling affected by your/his/her participation in the treatment?

9. Was your/his/her participation in the treatment affected by your/his/her gambling?

10. In what way could the treatment have been designed to increase the chances for you/him/her to continue the treatment? /What type of treatment do you think would have suited you/him/her?

11. Approximately $30-50 \%$ of participants of any given PG treatment drop out, what do you think is the explanation for that?

12. What type of treatment/support do you think a problem gambler need?

13. What type of treatment/support do you think a CSO of a problem gambler need? *

14. What did your CSO think about you discontinuing the treatment? $* *$

15. Have you tried any other treatments after this treatment?

a. What were the results of that?

16. Has he/she tried any other treatments after this treatment?

a. What were the results of that?

17. Is there anything else you would like to add?

\footnotetext{
* Only posed to CSOs

** Only posed to problem gambler
} 
live in Sweden, speak, read and write Swedish, and display no signs of more severe psychiatric conditions requiring further treatment.

\section{Participants}

The 16 participants consisted of eight individuals with gambling problems who had dropped out of treatment, and eight CSOs who were CSOs to gamblers who had dropped out of treatment. The CSOs were all related to a gambler who had dropped out, but the CSOs participated independently of whether the gambler wanted to participate in this study or not. Drop-out was defined as having completed half of the treatment modules or less, which has been referred to as an example of "non-usage attrition" in research regarding Internet-based interventions (Eysenbach, 2005), referring to when participants do not participate in the intervention. Most participants had dropped out after the first module (see Table 2), but in order to make it possible to capture themes related to the given PG interventions, we decided to include everyone who participated in half of the treatment or less.

Of 136 included individuals with gambling problems in the RCT, 46 participants, or $33.8 \%$, completed less than half of the treatment. Of these 46,16 had either stated the reasons for their withdrawal and/or stated that they had no wish to be contacted by the study team. For those that stated their reason for withdrawal, the reasons differed; a few stated they were no longer in need of treatment, others had commenced other treatment options, and a few stated they didn't have time to participate in treatment. We attempted to contact all of the remaining 30 , but 13 had either changed numbers or did not respond, while nine either declined participation or hung up. The remaining eight gamblers were included in the study, see Table 2 for details. Two of the CSOs and two of the gamblers had been participating in the study together, but they were interviewed separately. The included participants had a mean score on Problem Gambling Severity Index (PGSI) of $19.93(\mathrm{SD}=5.57)$ at pre-treatment? compared to 20.3 (SD = 4.22) for all RCT participants. PGSI has a score of $0-27$, where a score above 8 indicates gambling problems (Ferris \& Wynne, 2001).

\section{Analytical Approach}

A thematic analytical approach was used, following the six steps proposed by Braun and Clarke (2006) (Braun \& Clarke, 2006): familiarizing yourself with the data, generation initial codes, searching for themes, reviewing themes, defining and naming themes and producing the report. The interviews were transcribed by Author 1, and everything that was related to the questions, including laughs and pauses, was included in the transcription. Parts of the interviews were omitted from the transcription, i.e., small talk that contained information unrelated to the research topic.

In the first stage of analysis, a template for generating codes was created. Author 1 and Author 2 coded three of the transcriptions independently of each other by going through the transcripts, highlighting significant units and
Table 2 Description of included participants

\begin{tabular}{clllll}
\hline & Gender & $\begin{array}{l}\text { Age during } \\
\text { interview }\end{array}$ & $\begin{array}{l}\text { Relationship to CSO/ } \\
\text { gambler }\end{array}$ & $\begin{array}{l}\text { Completed mod- } \\
\text { ules* }\end{array}$ & Treatment arm \\
\hline $\begin{array}{c}\text { Gamblers } \\
\text { T1 }\end{array}$ & M & 27 & Partner & 2 & BCT \\
T2 & M & 34 & Child & 0 & BCT \\
T3 & M & 23 & Child & 0 & CBT \\
T4 & F & 53 & Friend/colleague & 2 & CBT \\
T5 & F & 58 & Partner & 1 & CBT \\
T6 & M & 40 & Child & 1 & BCT \\
T7 & M & 25 & Child & 1 & CBT \\
T8 & M & 33 & Partner & 0 & CBT \\
CSOs & & & & & BCT \\
T9 & F & 63 & Parent & 1 & CBT \\
T10 & F & 55 & Parent & 0 & CBT \\
T11 & F & 51 & Partner & 0 & CBT \\
T12 & M & 60 & Partner & 1 & BCT \\
T13 & M & 62 & Parent & 1 & CBT \\
T14 & F & 56 & Parent & 1 & BCT \\
T15 & F & 30 & Partner & 3 & BCT \\
T16 & F & 43 & Sister & 5 & \\
\hline
\end{tabular}

${ }^{*}$ The number of completed modules to completed modules for the gambler 
creating preliminary codes. A fourth transcription was coded together, and was subsequently coded by Author 3 to create a common code template, before Author 1 coded the remaining transcriptions. All codes were noted in the margins of the transcription and consisted of short sentences such as "dissatisfied with treatment" or "drop-out unrelated to treatment". Throughout this process, the authors read and re-read the transcriptions to assure that the new codes adequately reflected the content in the transcriptions.

The codes were then analyzed to identify possible themes and subthemes. This was a collaborative effort, and the authors regularly met to discuss the interpretation of the text. Up until this part of the process, answers from CSOs and gamblers had been handled separately. At this stage, based on the structure and content of the gathered data, all answers were organized within the same themes and sub-themes. The themes and sub-themes identified were grouped according to what research question they primarily belonged to, but the underlying codes could have materialized as responses to questions referring to the other research questions (e.g., an answer regarding reasons for drop-out could have been made on a question on recovery).

During the analytical process, the themes and the subthemes were reviewed several times and changed according to the discussion among the authors, and as the understanding of the material became more refined. Lastly, the themes were defined and named, before producing this article.

\section{Results}

The results were divided into three overarching themes that represent the process of staying in treatment and to recovery in PG: obstacles to stay in treatment, both facilitating and impeding factors to stay in treatment and recovery and facilitators to stay in treatment and recover. Obstacles to stay in treatment includes subthemes on processes that tend to impede positive changes and that frequently precede drop-out from treatment. Both facilitating and impeding factors for treatment and recovery highlights processes that could either be helpful or impede the process of change, depending on the context. Facilitators of change consists of processes identified by the participants as helpful in the process of change. What can be summarized throughout all these themes, is that most aspects of recovery - the treatment seeking process, participation in treatment and later attempts to change one's gambling - had been characterized by alternating periods of progress and setbacks. Across themes, CSOs and individuals with gambling problems' accounts were generally consistent, but CSOs tended to highlight negative emotions and life circumstances as important factors explaining relapses and drop-out from treatment more than individuals with gambling problems. The individuals with gambling problems often spontaneously mentioned support from others in the form of peer support as an important facilitator to change, while this was not mentioned by any $\mathrm{CSO}$. There were also some discrepancies regarding CSO involvement, where CSOs generally stressed its importance, while the individuals with gambling problems tended to be more reluctant.

\section{Obstacles to Stay in Treatment and Recovery}

Several obstacles to treatment continuation and recovery were identified.

\section{Relapse \& Increase of Negative Emotions - Intertwined Processes}

Relapse was often mentioned as a reason for drop-out from treatment. Relapses seem to create negative emotions and thoughts about one's capability to change, hopelessness, as well as to the value of the treatment itself. Negative emotions/mood caused by the relapse or other life-circumstances were mentioned as reasons to drop out and hinder recovery. The causal order was not clear to participants, and many described experiencing these processes simultaneously. Participants described these experiences were unbearable and increased impulses of avoidance. Motivation was also tightly connected to negative mood, both as preceding negative mood, but also as a result of negative mood, and could change quickly.

"Well, I started to gamble again a little, and then it became very stressful for me when I got questions about (gambling), and I know I just rushed through certain (treatment modules), just "click-click-click", just to make it go away. And these modules...I would have to access them and feel things, which I couldn't handle, so then I didn't reply to them." Female gambler (T4).

"I know I was feeling very low during this period. It might have been that it (recovering from $P G$ ) was too much of an obstacle, yes, it was that kind of period in life. Life felt hopeless, and, yeah, I think it (the gambling) came and went. [...] It's pretty easy to stay away from gambling when everything else is fine, and everything works as it should. But, well, life is often the opposite, and then when you start (gambling) again, it's hard to stop." Male gambler (T6).

\section{The Difficulty of Committing To Treatment \& the Impact Or The Surrounding Context}

To some participants the experience of seeking and committing to treatment was a difficult process, and sometimes 
causing resistance to change, which contributed to later drop-out. Some individuals with gambling problems seemed to have mixed feelings about many aspects of the treatments they had participated in, to the involvement of CSOs, as well as to their own gambling, causing ambivalence.

"I buried my head in the sand, because I didn't want to accept that it (the gambling) was a problem. But at the same time, I applied for help again. I guess I was pretty ambivalent". Female gambler (T5).

Many individuals with gambling problems described a tendency to try different treatments and support options without committing to any of them. Typically, participants had signed up to several treatments over the years, only to subsequently drop out of them. Signing up for new treatments were, for some, an act of hope and move forward, but could rapidly instead been perceived as a burden and lack of autonomy to decide how to move forward. Furthermore, to some, the commitment to treatment were connected to life circumstances. When such life circumstances changed, such as divorce, change of medication or lack of Internet access, some chose to drop-out. The financial consequences of the gambling, such as being unable to pay phone bills or for the Internet connection, was also mentioned as an obstacle to participate in treatment.

\section{Both Facilitating \& Impeding Factors}

The theme of both facilitating and impeding factors shows how some processes could be both helpful and impeding recovery and staying in treatment, depending on the context.

\section{Content \& Format of Treatment}

Some participants mentioned some well-known advantages of Internet-based treatment, such as lowering physical and mental barriers to psychological treatments. It was for many described as a first step, and the only potential step to take at the time.

"I think that, for the part of my boyfriend, he probably couldn't have taken a step as big as actually talking to someone in flesh and blood. So, it was kind of, at least for him, a smaller step to take [enrolling in the RCT] than to seek ordinary care." Female CSO, girlfriend of problem gambler (T15).

"That it could be this simple and easy! I was very shameful, and to not go out and say...because I wasn't ready to seek any other treatment at that point. It helped me a lot to gain insight and that I got some support. It was a relief, otherwise I don't know what would have happened." Female gambler, (T4).
Some pointed out that the lower barriers into treatment also meant lower barriers to drop out of treatment. Most participants had limited experience of the treatment offered in the RCT, since they dropped out prematurely. Nevertheless, some participants expressed disbelief in Internet-based treatments and preferred a face-to-face contact in treatment. Moreover, several of them had previous and later experiences from other treatment options. Few expressed any clear preferences for specific treatment components (e.g., cognitive restructuring, exposure therapy, communication training). Others claimed they were unaware they had dropped out of treatment, confusing follow up measures with the actual treatment content:

NN: "How come you didn't finish the program?" Male gambler (T8): "No! I thought I had finished it! [laughter] I can tell you that. But the e-mails just stopped coming, and then there was one after some months, another one after a year, and then nothing. It was the same for my wife (who participated as CSO)." Male gambler (T8).

\section{The Impact of Whom, Problem Gambler or CSO, Took the Measures to Change}

CSOs tended to be the ones who took measures to change the gambling in various ways. For some individuals with gambling problems, this was seen as helpful, while others felt they had no options:

"Eh, partly I wanted to quit myself actually. But, the, well, my partner, my ex, shit, she freaked out. So, then she forced me (to seek treatment)." Male gambler (T1).

"It was actually my mom who showed it to me, and then I said "absolutely"." Male gambler (T6).

One reason for drop-out included relatively positive or neutral emotions and behaviors, captured by the emotional recovery sub-theme. Some participants stated that recovery, and a more stable emotional state, were factors contributing to a perceived lack of need for further treatment. This led to participants dropping out of the treatment:

"My son had an incredibly tough time and used gambling as an escape route, and then it (the gambling) escalated. But when his life got back on track, then he didn't have any need to gamble again". Female CSO, mother of problem gambler (T10).

"This time I didn't feel I needed it (the treatment) anymore. I had stopped gambling. Yeah, I felt like I didn't need it. In the beginning, I think I participated for a couple of weeks perhaps. But then it sort of fizzled out." Male gambler (T1).

"He went through big changes in life at that time, with a divorce and a new relationship, and as far as 
I know he has had no tendencies to relapse since. A lot changed thanks to that (---) I didn't know he didn't finish (the treatment), but I imagine he didn't feel the need for it, when it (the gambling problems) wasn't as urgent anymore." Female CSO, sister of a problem gambler (T16).

\section{Facilitators of Change}

The results in the facilitators of change theme identifies what the participants experienced as helpful in terms of recovery and to stay in treatment, and to some extent what was important for them in any given PG treatment.

\section{The Importance of the First Steps}

To some participants, participation in this study, albeit very limited, might have played an important role in recovery. Several participants mentioned participating in the study as either one of several important contributing factors to recovery, or as something that over time had influenced them to change their gambling behavior.

"We would have tried to get out of it anyway. But
this was some help on the way, it was like one more
reminder (to try to change). (---) It's like this with
everything in this world, it's never just one thing that
helps. Many things need to go in the right direction,
and they need to do it often." Male CSO, husband of
problem gambler (T11).

\section{The Key of Openness \& Support}

A large portion of both gamblers and CSOs described openness as a highly important aspect of recovery. Several participants highlighted the importance of being honest about problems towards CSOs, and a deeper understanding of one's own behavior, and its consequences, as well as recognizing oneself in others with similar experiences.

"It's all about admitting your addiction I think, to yourself."

Female gambler, (T4).

“- It's (the treatment) something you did together and share, and you get more of an understanding of each other and what the other person thinks and so on." Male gambler, (T8)

For many individuals with gambling problems, it was important to get support from CSOs, therapists, and peers with similar experiences in order to recover from PG. It was particularly evident that peer support filled an important function for many gamblers. Sometimes this support seemed to be limited to hearing others describing similar experiences of PG, but it was nonetheless important for recovery.

"You need someone with some experience of what can happen and so on. You probably don't need that much support from someone who's never gambled. But (you need support from) someone who's had a rough time, and who got back up again, and who's fought, and who knows that gambling is a dead end." Male gambler (T2).

"I think it (involving a CSO in treatment) was a prerequisite for me to succeed the way I did." Female gambler, (T4).

\section{Discussion}

This study aimed to investigate the reasons for dropping out of PG treatment and the path to recovery. The findings of this study illustrates that many aspects related to PG and the ambition to recover from PG was characterized by alternating periods of progress and setbacks. This is similar to earlier mentioned findings in other qualitative studies on PG recovery and treatment engagement (Pickering et al., 2020a, b; Anderson et al., 2009b; Nixon \& Solowoniuk, 2006). Furthermore, the individuals with gambling problems' own accounts of this process often provide some contradicting statements, indicating that they had conflicting thoughts and emotions about many aspects of their PG. For example, some participants highlighted both the advantage of involving CSOs in treatment, and at the same time stated that they preferred dealing with problems without CSO involvement. One reason for the somewhat conflicting images is that the recovery process generally takes time, and during that process a problem gambler is faced with different, and interchangeable, types of situations and emotions. Over a period of time, the problem gambler might experience a wide array of emotions and hold different viewpoints, e.g., initially have a positive view of CSO involvement, but over time hold a more negative view. Thus, the subthemes identified in this study must be understood as a whole, rather than as separate factors that are added to increase or decrease the odds of remaining in treatment or recovering from PG.

Ambivalence is common in problematic use disorders, where attempts to change one's behavior are replaced by periods of problem denial, or disillusion about chances of recovery, and continued substance use or gambling. This is also seen in relationship to CSOs and their involvement in treatment, as well as in the tendency to hop on and off various treatments, seemingly regardless of the treatment content. Some CSOs also stressed what they perceived as a lack of readiness to commit to change among the individuals with gambling problems. But ambivalence also 
likely stems from the many experiences of failed attempts to quit, and relapses highlighted in this study. The theme regarding the intertwined nature of relapses and negative emotions highlighted how feelings of hopelessness and shame characterized many participants' emotional state when relapsing and dropping out. A large body of research confirms that individuals with gambling problems tend to have comorbid disorders, such as anxiety and depression (Lorains et al., 2011). This was also indicated in other papers from this study (Nilsson et al., 2018; Nilsson et al., 2020), as well as the tendency for participants that dropped out to have higher levels of comorbidity in general Nilsson et al., 2018). Some participants' accounts of their behavior when participating in the treatment, could perhaps be identified as experiential avoidance, i.e., the unwillingness to remain in contact with negative internal states, such as thoughts and emotions. These are well-known processes in CBT conceptualizations of psychological distress (Chawla \& Ostafin, 2007; Hayes et al., 1996). This suggests that issues of comorbidity and negative emotions should be taken into consideration when designing intervention studies and providing treatment.

It also became apparent that many factors of the participants' general life circumstances contributed to drop-out. Some were clearly linked to the problem gambling itself, such as lacking funds to pay the telephone bill and thus being inaccessible to the therapist or unable to log on to the treatment platform. Other factors contributing to drop-out such as divorce, changed working hours or new medication were not as clearly linked to PG, but could instead be understood as factors contributing to recovery (Cunningham et al., 2009). However, one speculation is that the underlying factors identified in earlier studies to predict drop-out, e.g. impulsivity or personality traits (Melville et al., 2007), might partly explain both PG and life circumstances linked to drop-out.

The treatment format itself might also have contributed to the drop-outs, which is a well-known challenge with Internet-based interventions (Melville et al., 2010; Christensen et al., 2009; Eysenbach, 2005). Studies investigating why clients seek Internet-based treatments for PG highlight the importance of anonymity and flexibility of the format compared to face-to-face treatments (Rodda, Lubman, Dowling, \& McCann, 2013a, b; Wood \& Wood, 2009). This could improve the availability of the treatment, and thus reach clients who would otherwise not have participated in formal treatment. On the other hand, potential clients who prefer a higher degree of personal interaction, or who have less digital literacy might be reluctant to seek Internet-based interventions. This could create a sampling bias, but studies on who is attracted by Internet-based interventions have yielded mixed results regarding what factors characterize those who seek treatment online. Among the factors identified are higher levels of psychological distress, higher level of education, participation in prior treatment, and being female (Crisp \& Griffiths, 2014; Donkin et al., 2012; Ryan et al., 2010), as well as no significant differences from those seeking face-to-face treatment (Klein \& Cook, 2010).

Participants in this study praised the low threshold into treatment, but the threshold out of treatment could also have been lower compared to face-to-face treatments. Interestingly, some participants were seemingly unaware that they had dropped out of treatment, confusing follow-up measurements with the treatment itself, which highlights the importance of carefully explaining the rationale, format and outline of treatments. This could be understood through the concept of health literacy (Nutbeam, 2008), where individuals vary in their ability to understand crucial aspects of any given treatment.

Some participants claimed that they had dropped out of treatment because they did not feel the need for treatment any longer. This is in line with previous findings that some gamblers quit treatment because they believe they have recovered (Dunn et al., 2012), which may or may not be an accurate self-evaluation. This is also in line with research on natural recovery in PG (Slutske, 2014), as well as findings that outcomes from brief interventions might be on par with longer treatments for PG (Hodgins et al., 2009; Quilty et al., 2019). Many individuals with gambling problems also seek treatment in a state of crisis (Evans \& Delfabbro, 2005), which could have changed quite substantially at the time when treatment actually begins, making further participation seem unnecessary. At the same time, this is also an illustration of the often unstable path to recovery, where changes can be fast in both negative and positive directions. There is also a risk that individuals with gambling problems downplay the risks of future relapses or setbacks.

In sum, drop-out from PG treatment has to be seen in light of the complex characteristics of PG, where comorbid disorders, relapses, life circumstances, ambivalence and the PG itself interact to cause both help-seeking and drop-out from treatment. The treatment format and the content of the treatment might also affect the number of participants who drop out of treatment. In general, these processes leading to drop-out reflects the overarching theme of how recovery from PG is composed by an unstable and unpredictable path.

As for factors perceived to facilitate recovery, some participants saw a strict and rigorous therapist as important for recovery. This is also underscored by research on the efficacy of Internet-based interventions, where strict deadlines is positively correlated with positive outcomes of treatment (Paxling et al., 2013). However, as became evident, CSOs often took the role of pushing gamblers in to treatment, and aimed to use control measures, such as overseeing or controlling the gambler's economy. Previous research has suggested that there is a risk that CSOs' strive for greater 
control over the gambling might actually provoke feelings of an increased need to gamble for some individuals with gambling problems (Bertrand et al., 2008), which indicates that such pressure is not always beneficial, but might in the worst case aggravate the PG. As for this study, it is clear that CSOs played an important role in motivating, or sometimes coercing, gamblers to seek treatment, but also that they played an important role in recovery. This is in line with findings from studies on alcohol dependence where social support has been identified as a key in recovery (Bischof et al., 2003, 2007), and for some trials involving CSOs in the treatment of PG (Ingle et al., 2008), albeit not the RCT this study is based on [insert reference]. Among the individuals with gambling problems, some would likely not have entered treatment without a push from a CSO, but some might have dropped out as a result of feeling pressured into treatment.

Peer support from people with similar experiences of problem gambling was also seen as highly important for those gamblers who had experienced it. Participants generally pointed to important insights gained from this type of support, as well as how stories from others helped to relieve them of feelings of guilt and shame. Peer support emerged as a theme, even though it was not explicitly mentioned in the interview guide, but instead spontaneously brought up for discussion by several participants. It is noteworthy that while peer support for PG is common, it is rarely a feature of treatment protocols in intervention studies or regular health care, nor is it focus of much research interest.

All participants self-identified as recovered from PG at the time of the interviews, as far as was communicated in the interviews. It seemed like participation in the study had some positive effect on the gambler, even though he or she dropped out prematurely. This very brief involvement in the study was likely one of several factors contributing to recovery. It indicates that processes inherent to the treatment seeking process, such as committing to change, disclosing one's PG, or reflecting on money lost to gambling are likely important drivers of change in PG. This is similar to previously mentioned findings on emotional recovery and how brief interventions seem to suffice to assist certain individuals with gambling problems to recovery (Quilty et al., 2019).

One often discussed question in PG research is when recovery from PG actually occurs, and how to measure it (Nower \& Blaszczynski, 2008; Pickering et al., 2018, 2020a, b). While beyond the scope of this article, the key of openness and support subtheme might represent part of the answer. Expressions like "insight" and "honesty" were used to describe a previously missing sense of openness, acceptance, responsibility and understanding of the PG. It could be argued that when the type of openness described by the participants has become the norm, individuals with gambling problems are at a stage when they have recovered from PG, and to some extent has "left" the unstable path. Other studies have identified similar concepts such as a shared narrative (Nuske \& Hing, 2013) insight (Pickering et al., 2020a, b) and recovery wisdom (Pickering et al., 2020a, b) as critical facets of recovery in PG. The statements given regarding peer support, where participants highlighted the importance of seeing oneself in others with similar experiences, as well as getting appropriate support from peers and CSOs, also give an insight into what processes can be important in recovery from PG.

As mentioned, one observation was that essentially all participants, as well as former individuals with gambling problems described by CSOs, self-identified as having recovered from their PG. This possibly depends on a selection bias, which limits what conclusions that can be drawn from the result, and it was not verified with any psychological tests or clinical assessment. Nevertheless, it does show that it is possible to recover despite following an unstable path. Speculatively, being recovered from PG could have provided participants with a greater ability to reflect on processes and factors influencing PG, treatment seeking, and recovery than if they had still struggled with ongoing PG.

The results of this study give some suggestions for future research and development of PG interventions. In our view, this could be applicable for PG interventions in general, even though the study stems from an Internet-based intervention. The results suggest that there is reason to consider a holistic view of the individual's situation, in order to minimize drop-out from PG treatment. Negative emotions, comorbidities and challenging life circumstances tend to interfere with treatment participation, and should be considered as additional focuses for treatment. Relatively novel treatment approaches such as mindfulness based interventions (Chen et al., 2014) and emotional regulation therapy (Zargar et al., 2019) could provide part of the answer. Similarly, definitions of recovery from PG should perhaps incorporate a wider assessment of the participants' well-being rather than focusing narrowly on abstinence from gambling or controlled gambling, in line with the suggestions made by Pickering et al., (2020a, b). Lastly, future research should further investigate the possible role peer support could have in formal PG treatment, since it was a much wished-for treatment component among several participants in this study.

\section{Limitations}

One limitation to this study is that we were unable to reach many of the participants that had dropped out of treatment. It is unknown whether their experience would differ in relation to those included in this study. Furthermore, this study investigates drop-out and recovery in the context of Internet-based treatment and it is not certain the results from this study could be generalized to those dropping out of face-to-face treatments. 
This design of this study did not allow us to tie the definition and timing of dropout to concrete measures of changes in gambling behavior or other dimensions of recovery. Another limitation is that the interviews were conducted over telephone, which makes it difficult to control for possible disturbances for the person being interviewed, potentially impacting the quality and depth of data. The interviews were conducted at least two years after the participants dropped out of treatment, which could make it more difficult to accurately recall events and emotions, compared to if the interviews had been made in conjunction with the treatment. Nevertheless, this offers opportunities for a broader perspective on recovery. A final limitation is that the interviews were conducted in Swedish, while the results are described in English, and some of the nuances could have been lost in translation.

\section{Conclusions}

The results of this study gave new insights, and corroborated some earlier found factors, about recovery and drop out from PG treatment, but the most important theme was that drop outs is part of a larger theme. The overarching unstable path to recovery shows how multiple aspects of recovery among the participants in this study - treatment seeking, involving CSOs, attending treatment, behavior change - was marked by alternating periods of progress and setbacks. Specific subthemes regarding negative emotions and its' role in relapses highlighted possible considerations when designing PG interventions, e.g., working with comorbidity and experiential avoidance.

Funding Open access funding provided by Karolinska Institute. This work was supported by Svenska Spel's Independent Research Council (grant number 2013-0015). It had no influence over the design, outcomes or analysis of this study. Svenska Spel is the state-organized gambling provider in Sweden, and it sets aside money to support research on PG. Its research committee is independent from the main organization, and the research is conducted according to regular university standards.

Data Availability The data that support the findings of this study are available on request from the corresponding author. The data are not publicly available due to data containing information that could compromise the privacy of research participants.

\section{Declarations}

Conflicts of Interest No conflicting or competing interests reported.

Ethical Approval The regional ethics board of Stockholm, Sweden, number 2014/175-31/5.

Open Access This article is licensed under a Creative Commons Attribution 4.0 International License, which permits use, sharing, adaptation, distribution and reproduction in any medium or format, as long as you give appropriate credit to the original author(s) and the source, provide a link to the Creative Commons licence, and indicate if changes were made. The images or other third party material in this article are included in the article's Creative Commons licence, unless indicated otherwise in a credit line to the material. If material is not included in the article's Creative Commons licence and your intended use is not permitted by statutory regulation or exceeds the permitted use, you will need to obtain permission directly from the copyright holder. To view a copy of this licence, visit http://creativecommons.org/licenses/by/4.0/.

\section{References}

Anderson, S., Dobbie, F., \& Reith, G. (2009). Recovery from problem gambling: A qualitative study. Edinburgh, UK: Scottish Centre for Social Research.

Anderson, S., Dobbie, F., \& Reith, G. (2009). Recovery from problem gambling: A qualitative study. Edinburgh, UK: Scottish Centre for Social Research.

Aragay, N., Jiménez-Murcia, S., Granero, R., Fernández-Aranda, F., Ramos-Grille, I., Cardona, S., Garrido, G., Islam, M. A., Menchón, J. M., \& Vallès, V. (2015). Pathological gambling: Understanding relapses and dropouts. Comprehensive Psychiatry, 57, 58-64.

Bertrand, K., Dufour, M., Wright, J., \& Lasnier, B. (2008). Adapted Couple Therapy (ACT) for pathological gamblers: A promising avenue. Journal of Gambling Studies, 24, 393-409.

Bischof, G., Rumpf, H.-J., Hapke, U., Meyer, C., \& John, U. (2003). Types of natural recovery from alcohol dependence: A cluster analytic approach. Addiction, 98, 1737-1746.

Bischof, G., Rumpf, H.-J., Meyer, C., Hapke, U., \& John, U. (2007). Stability of subtypes of natural recovery from alcohol dependence after two years. Addiction, 102, 904-908.

Braun, V., \& Clarke, V. (2006). Using thematic analysis in psychology. Qualitative Research in Psychology, 3, 77-101.

Brorson, H. H., Arnevik, E. A., Rand-Hendriksen, K., \& Duckert, F. (2013). Drop-out from addiction treatment: A systematic review of risk factors. Clinical Psychology Review, 33, 1010-1024.

Brown, R. I. F. (1986). Dropouts and continuers in Gamblers Anonymous: Life-context and other factors. Journal of Gambling Behavior, 2, 130-140.

Chawla, N., \& Ostafin, B. (2007). Experiential avoidance as a functional dimensional approach to psychopathology: An empirical review. Journal of Clinical Psychology, 63, 871-890.

Chen, P., Jindani, F., Perry, J., \& Turner, N. L. (2014). "Mindfulness and problem gambling treatment", Asian Journal of Gambling Issues and Public. Health, 4, 1-17.

Christensen, H., Griffiths, K. M., \& Farrer, L. (2009). Adherence in internet interventions for anxiety and depression. Journal of medical Internet research, 11(2), e13. https://doi.org/10.2196/ jmir.1194

Cowlishaw, S., Merkouris, S., Dowling, N., Anderson, C., Jackson, A., \& Thomas, S. (2012). Psychological therapies for pathological and problem gambling. The Cochrane database of systematic reviews, 11, CD008937. https://doi.org/10.1002/14651858. CD008937.pub2

Crisp, D. A., \& Griffiths, K. M. (2014). Participating in online mental health interventions: who is most likely to sign up and why? Depression research and treatment, 2014, 790457. https://doi. org/10.1155/2014/790457

Cunningham, J. A., Hodgins, D. C., \& Toneatto, T. (2009). Natural history of gambling problems: Results from a general population survey. Sucht, 55, 98-103.

Donkin, L., Hickie, I. B., Christensen, H., Naismith, S. L., Neal, B., Cockayne, N. L., \& Glozier, N. (2012). Sampling bias in an 
internet treatment trial for depression. Translational Psychiatry, 2, e174-e274.

Dowling, N. (2009). Client characteristics associated with treatment attrition and outcome in female pathological gambling. Addiction Research \& Theory, 17, 205-219.

Dunn, K., Delfabbro, P., \& Harvey, P. (2012). A preliminary, qualitative exploration of the influences associated with drop-out from cognitive-behavioural therapy for problem gambling: An Australian perspective. Journal of Gambling Studies, 28, 253-272.

Evans, L., \& Delfabbro, P. H. (2005). Motivators for change and barriers to help-seeking in Australian problem gamblers. Journal of Gambling Studies, 21, 133-155.

Eysenbach G. (2005). The law of attrition. Journal of medical Internet research, 7(1), e11. https://doi.org/10.2196/jmir.7.1.e11

Ferris, J. A., \& Wynne, H. J. (2001). The Canadian problem gambling index (pp. 1-59). Ottawa, ON: Canadian Centre on Substance Abuse.

Goodwin, B. C., Browne, M., Rockloff, M., \& Rose, J. (2017). A typical problem gambler affects six others. International Gambling Studies, 17, 276-289.

Grant, J. E., Kim, S. W., \& Kuskowski, M. (2004). Retrospective review of treatment retention in pathological gambling. Comprehensive Psychiatry, 45, 83-87.

Grant, J. E., Williams, K. A., \& Kim, S. W. (2006). Update on pathological gambling. Current Psychiatry Reports, 8, 53-58.

Hayes, S. C., Wilson, K. G., Gifford, E. V., Follette, V. M., \& Strosahl, K. (1996). Experiential avoidance and behavioral disorders: A functional dimensional approach to diagnosis and treatment. Journal of Consulting and Clinical Psychology, 64, 1152

Hodgins, D. C., Currie, S. R., Currie, G., \& Fick, G. H. (2009). Randomized trial of brief motivational treatments for pathological gamblers: More is not necessarily better. Journal of Consulting and Clinical Psychology, 77, 950.

Hodgins, D. C., \& N. el-Guebaly. . (2000). Natural and treatmentassisted recovery from gambling problems: A comparison of resolved and active gamblers. Addiction, 95, 777-789.

Hodgins, D. C., Stea, J. N., \& Grant, J. E. (2011). Gambling disorders. The Lancet, 378, 1874-1884.

Ingle, P. J., Marotta, J., McMillan, G., \& Wisdom, J. P. (2008). Significant others and gambling treatment outcomes. Journal of Gambling Studies, 24, 381-392.

Jüni, P., Altman, D. G., \& Egger, M. (2001). Assessing the quality of controlled clinical trials. BMJ, 323, 42-46.

Kalischuk, R. G., Nowatzki, N., Cardwell, K., Klein, K., \& Solowoniuk, J. (2006). Problem gambling and its impact on families: A literature review. International Gambling Studies, 6, 31-60.

Klein, B., \& Cook, S. (2010). Preferences for e-mental health services amongst an online Australian sample. E-Journal of Applied Psychology, 6(1).

Lorains, F. K., Cowlishaw, S., \& Thomas, S. A. (2011). Prevalence of comorbid disorders in problem and pathological gambling: Systematic review and meta-analysis of population surveys. Addiction, 106, 490-498.

Melville, K. M., Casey, L. M., \& Kavanagh, D. J. (2007). Psychological treatment dropout among pathological gamblers. Clinical Psychology Review, 27, 944-958.

Melville, K. M., Casey, L. M., \& Kavanagh, D. J. (2010). Dropout from Internet-based treatment for psychological disorders. British Journal of Clinical Psychology, 49, 455-471.

Neal, P., Delfabbro, P., \& O'Neil, M. (2005). Problem gambling and harm: A national definition. Adelaide, Australia: South Australia Centre for Economic Studies.

Nixon, G., \& Solowoniuk, J. (2006). An insider's look into the process of recovering from pathological gambling disorder: An existential phenomenological inquiry. International Journal of Mental Health and Addiction, 4, 119-132.
Nower, L., \& Blaszczynski, A. (2008). Recovery in pathological gambling: An imprecise concept. Substance Use \& Misuse, 43, 1844-1864.

Nuske, E., \& Hing, N. (2013). A narrative analysis of help-seeking behaviour and critical change points for recovering problem gamblers: The power of storytelling. Australian Social Work, 66, 39-55.

Nutbeam, D. (2008). The evolving concept of health literacy. Social Science \& Medicine, 67, 2072-2078.

Paxling, B., Lundgren, S., Norman, A., Almlöv, J., Carlbring, P., Cuijpers, P., \& Andersson, G. (2013). Therapist behaviours in internet-delivered cognitive behaviour therapy: Analyses of e-mail correspondence in the treatment of generalized anxiety disorder. Behavioural and Cognitive Psychotherapy, 41, 280-289.

Pelletier, O., Ladouceur, R., \& Rhéaume, J. (2008). Personality disorders and pathological gambling: Comorbidity and treatment dropout predictors. International Gambling Studies, 8, 299-313.

Pfund, Rory A, Samuel C Peter, Nicholas W McAfee, Meredith K Ginley, James P Whelan, and Andrew W Meyers. 2021. 'Dropout from face-to-face, multi-session psychological treatments for problem and disordered gambling: A systematic review and meta-analysis', Psychology of Addictive Behaviors

Pfund, R. A., Peter, S. C., Whelan, J. P., \& Meyers, A. W. (2018) 'When does premature treatment termination occur? Examining Session-by-Session Dropout among Clients with Gambling Disorder', Journal of Gambling Studies, 34, 617-630.

Pickering, D., Blaszczynski, A., \& Gainsbury, S. M. (2021). Development and psychometric evaluation of the Recovery Index for Gambling Disorder (RIGD). Psychology of addictive behaviors : journal of the Society of Psychologists in Addictive Behaviors, 35(4), 472-485. https://doi.org/10.1037/adb0000676

Pickering, D., Keen, B., Entwistle, G., \& Blaszczynski, A. (2018). Measuring treatment outcomes in gambling disorders: A systematic review. Addiction, 113, 411-426.

Pickering, D., Spoelma, M. J., Dawczyk, A., Gainsbury, S. M., \& Blaszczynski, A. (2020b). 'What does it mean to recover from a gambling disorder? Perspectives of Gambling Help Service Users', Addiction Research \& Theory, 28, 132-143.

Potenza, M. N., Balodis, I. M., Derevensky, J., Grant, J. E., Petry, N. M., Verdejo-Garcia, A., \& Yip, S. W. (2019). Gambling disorder. Nature Reviews Disease Primers, 5, 1-21.

Quilty, Lena C., Wardell, Jeffrey D., Thiruchselvam, Thulasi, Keough, Matthew T., \& Hendershot, Christian S. (2019). Brief interventions for problem gambling: A meta-analysis. PLoS One, 14, e0214502.

Ramos-Grille, I., Gomà-i-Freixanet, M., Aragay, N., Valero, S., \& Vallès, V. (2015). Predicting treatment failure in pathological gambling: The role of personality traits. Addictive Behaviors, $43,54-59$

Rodda, Simone, Lubman, Dan I., Dowling, Nicki A., Bough, Anna, \& Jackson, Alun C. (2013). Web-based counseling for problem gambling: exploring motivations and recommendations. Journal of Medical Internet Research, 15, e99.

Rodda, S. N., Lubman, D. I., Dowling, N. A., \& McCann, T. V. (2013b). Reasons for using web-based counselling among family and friends impacted by problem gambling. Asian Journal of Gambling Issues and Public Health, 3, 1.

Ronzitti, S., Soldini, E., Smith, N., Clerici, M., \& Bowden-Jones, H. (2017). 'Gambling disorder: Exploring pre-treatment and intreatment dropout predictors. A UK Study', Journal of Gambling Studies, 33, 1277-1292.

Rossini-Dib, D., Fuentes, D., \& Tavares, H. (2015). A naturalistic study of recovering gamblers: What gets better and when they get better. Psychiatry Research, 227, 17-26.

Ryan, M. L., Shochet, I. M., \& Stallman, H. M. (2010). Universal online interventions might engage psychologically distressed 
university students who are unlikely to seek formal help. Advances in Mental Health, 9, 73-83.

Slutske W. S. (2006). Natural recovery and treatment-seeking in pathological gambling: results of two U.S. national surveys. The American journal of psychiatry, 163(2), 297-302. https:// doi.org/10.1176/appi.ajp.163.2.297

Smith, D., Harvey, P., Battersby, M., Pols, R., Oakes, J., \& Baigent, M. (2010). Treatment outcomes and predictors of drop out for problem gamblers in South Australia: A cohort study. Australian and New Zealand Journal of Psychiatry, 44, 911-920.

Statens Folkhälsoinstitut. (2010). Spel om pengar och spelproblem i Sverige 2008/2009. Huvudresultat från SWELOGS befolkningsstudie [SWELOGS, Swedish Longitudinal Gambling Study].

Swift, J. K., \& Greenberg, R. P. (2012). Premature discontinuation in adult psychotherapy: A meta-analysis. Journal of Consulting and Clinical Psychology, 80, 547.

Toneatto, T., Cunningham, J., Hodgins, D., Adams, M., Turner, N., \& Koski-Jannes, A. (2008). Recovery from problem gambling without formal treatment. Addiction Research \& Theory, 16, 111-120.

Vasiliadis, S., \& Thomas, A. (2018). Recovery agency and informal recovery pathways from gambling problems. International Journal of Mental Health and Addiction, 16, 874-887.

Westphal, J. R. (2007). Are the effects of gambling treatment overestimated? International Journal of Mental Health and Addiction, $5,65-79$.

Williams, R., Hann, R., Schopflocher, D., West, B., McLaughlin, P., White, N., King, K., \& Flexhaug, T. (2015). Quinte longitudinal study of gambling and problem gambling. Ontario Problem Gambling Research Centre.

Williams, R. J., Volberg, R. A., \& Stevens, R. M. (2012). The population prevalence of problem gambling: Methodological influences, standardized rates, jurisdictional differences, and worldwide trends. Ontario Problem Gambling Research Centre.
Wood, R. T., \& Wood, S. A. (2009). An evaluation of two United Kingdom online support forums designed to help people with gambling issues. Journal of Gambling Issues, (23), 5-30.

Yakovenko, I., \& Hodgins, D. C. (2016). Latest developments in treatment for disordered gambling: Review and critical evaluation of outcome studies. Current Addiction Reports, 3, 299-306.

Zargar, F., Bagheri, N., Tarrahi, M. J., \& Salehi, M. (2019). Effectiveness of emotion regulation group therapy on craving, emotion problems, and marital satisfaction in patients with substance use disorders: A randomized clinical trial. Iranian Journal of Psychiatry, 14, 283.

Nilsson, A., Magnusson, K., Carlbring, P., Andersson, G., \& Hellner, C. (2020). Behavioral couples therapy versus cognitive behavioral therapy for problem gambling: a randomized controlled trial. Addiction (Abingdon, England), 115(7), 1330-1342. https:// doi.org/10.1111/add.14900

Nilsson, A., Magnusson, K., Carlbring, P., Andersson, G., \& Hellner Gumpert, C. (2016). Effects of added involvement from concerned significant others in internet-delivered CBT treatments for problem gambling: study protocol for a randomised controlled trial. BMJ open, 6(9), e011974. https://doi.org/10.1136/ bmjopen-2016-011974

Nilsson, A., Magnusson, K., Carlbring, P., Andersson, G., \& Gumpert, C. H. (2018). The Development of an Internet-Based Treatment for Problem Gamblers and Concerned Significant Others: A Pilot Randomized Controlled Trial. Journal of gambling studies, 34(2), 539-559. https://doi.org/10.1007/s10899-017-9704-4

Publisher's Note Springer Nature remains neutral with regard to jurisdictional claims in published maps and institutional affiliations. 